\title{
SOME OPEN QUESTIONS ABOUT SYMPLECTIC 4-MANIFOLDS, SINGULAR PLANE CURVES, AND BRAID GROUP FACTORIZATIONS
}

\author{
DENIS AUROUX
}

\begin{abstract}
The topology of symplectic 4-manifolds is related to that of singular plane curves via the concept of branched covers. Thus, various classification problems concerning symplectic 4-manifolds can be reformulated as questions about singular plane curves. Moreover, using braid monodromy, these can in turn be reformulated in the language of braid group factorizations. While the results mentioned in this paper are not new, we hope that they will stimulate interest in these questions, which remain essentially wide open.
\end{abstract}

\section{INTRODUCTION}

An important problem in 4-manifold topology is to understand which manifolds carry symplectic structures (i.e., closed non-degenerate 2-forms), and to develop invariants that can distinguish symplectic manifolds. Additionally, one would like to understand to what extent the category of symplectic manifolds is richer than that of Kähler (or complex projective) manifolds. For example, one would like to identify a set of surgery operations that can be used to turn an arbitrary symplectic 4-manifold into a Kähler manifold, or two symplectic 4-manifolds with the same classical topological invariants (fundamental group, Chern numbers, ...) into each other.

Similar questions may be asked about singular curves inside, e.g., the complex projective plane. The two types of questions are related to each other via symplectic branched covers. A branched cover of a symplectic 4manifold with a (possibly singular) symplectic branch curve carries a natural symplectic structure. Conversely, every compact symplectic 4-manifold is a branched cover of $\mathbb{C P}^{2}$, with a branch curve presenting nodes (of both orientations) and complex cusps as its only singularities.

In the language of branch curves, the failure of most symplectic manifolds to admit integrable complex structures translates into the failure of most symplectic branch curves to be isotopic to complex curves. While the symplectic isotopy problem has a negative answer for plane curves with cusp and node singularities, it is interesting to investigate this failure more precisely. Various partial results have been obtained recently about situations where isotopy holds (for smooth curves; for curves of low degree), and

Partially supported by NSF grant DMS-0244844. 
about isotopy up to stabilization or regular homotopy. On the other hand, many known examples of non-isotopic curves can be understood in terms of braiding along Lagrangian annuli (or equivalently, Luttinger surgery of the branched covers), leading to some intriguing open questions about the topology of symplectic 4-manifolds versus that of Kähler surfaces.

If one prefers to adopt a more group theoretic point of view, it is possible to use braid monodromy techniques to reformulate these questions in terms of words in braid groups. For example, the classification of symplectic 4manifolds reduces in principle to a (hard) question about factorizations in the braid group, known as the Hurwitz problem.

In the following sections, we discuss these various questions and the connections between them, starting from the point of view of symplectic 4manifolds (in $\S 2$ ), then translating them in terms of plane branch curves (in $\S 3)$ and finally braid group factorizations (in $\S 4$ ).

\section{TOPOLOGICAL QUESTIONS ABOUT SYMPLECTIC 4-MANIFOLDS}

2.1. Classification of symplectic 4-manifolds. Recall that a symplectic manifold is a smooth manifold equipped with a 2 -form $\omega$ such that $d \omega=0$ and $\omega \wedge \cdots \wedge \omega$ is a volume form. The first examples of compact symplectic manifolds are compact oriented surfaces (taking $\omega$ to be an arbitrary area form), and the complex projective space $\mathbb{C P}^{n}$ (equipped with the FubiniStudy Kähler form). More generally, since any submanifold to which $\omega$ restricts non-degenerately inherits a symplectic structure, all complex projective manifolds are symplectic. However, the symplectic category is strictly larger than the complex projective category, as first evidenced by Thurston in 1976 31. In 1994 Gompf used the symplectic sum construction to prove that any finitely presented group can be realized as the fundamental group of a compact symplectic 4-manifold [15].

An important problem in symplectic topology is to understand the hierarchy formed by the three main classes of compact oriented 4-manifolds: (1) complex projective, (2) symplectic, and (3) smooth. Each class is a proper subset of the next one, and many obstructions and examples are known, but we are still very far from understanding what exactly causes a smooth 4-manifold to admit a symplectic structure, or a symplectic 4-manifold to admit an integrable complex structure.

One of the main motivations to study symplectic 4-manifolds is that they retain some (but not all) features of complex projective manifolds: for example the structure of their Seiberg-Witten invariants, which in both cases are non-zero and count embedded (pseudo)holomorphic curves [27, 28. At the same time, every compact oriented smooth 4 -manifold with $b_{2}^{+} \geq 1$ admits a "near-symplectic" structure, i.e. a closed 2-form which vanishes along a union of circles and is symplectic over the complement of its zero set [14, 18];

and it appears that some structural properties of symplectic manifolds carry over to the world of smooth 4-manifolds (see e.g. [29, 4]). 
Although the question of determining which smooth 4-manifolds admit symplectic structures and how many is definitely an essential one, it falls outside of the scope of this paper. Rather, our goal will be to obtain information on the richness of the symplectic category, especially when compared to the complex projective category.

We will restrict ourselves to the class of integral compact symplectic 4manifolds, i.e. we will assume that the cohomology class $[\omega] \in H^{2}(X, \mathbb{R})$ is the image of an element of $H^{2}(X, \mathbb{Z})$. This does not place any additional restrictions on the diffeomorphism type of $X$, but makes classification a discrete problem (by Moser's stability theorem, deformations that keep $[\omega]$ constant are induced by ambient isotopies).

By integrating the Chern classes of the tangent bundle and the symplectic class over the fundamental cycle $[X]$, one obtains various classical topological invariants: the Chern numbers $c_{1}^{2}(=2 \chi+3 \sigma)$ and $c_{2}(=\chi)$, the symplectic volume $[\omega]^{2}$, and $c_{1} \cdot[\omega]$. Hence, the first question we will ask is:

Question 2.1. Can one classify all integral compact symplectic 4-manifolds with given values of $\left(c_{1}^{2}, c_{2}, c_{1} \cdot[\omega],[\omega]^{2}\right)$ (and a given fundamental group)?

This question contains the geography problem, i.e. the question of determining which Chern numbers can be realized by compact symplectic 4manifolds. In some specific cases, Taubes' results on Seiberg-Witten invariants seriously constrain the list of possibilities. For example we have the following result [27]:

Theorem 2.2 (Taubes). Let $(X, \omega)$ be a compact symplectic 4-manifold with $b_{2}^{+} \geq 2$. Then $c_{1} \cdot[\omega] \leq 0$. Moreover, if $X$ is minimal (i.e. does not contain an embedded symplectic sphere of square -1$)$, then $c_{1}^{2} \geq 0$.

A lot is also known about the case $c_{1}^{2}=0$, from Seiberg-Witten theory and from various surgery constructions. For example, infinite families of simply connected symplectic 4-manifolds homeomorphic but not diffeomorphic to elliptic surfaces have been constructed (see e.g. 15, 10]). However, when $c_{1}^{2}>0$ very little is known, and many important questions remain open. For example it is unknown whether the Bogomolov-Miyaoka-Yau inequality $c_{1}^{2} \leq 3 c_{2}$, which constrains the Chern numbers of complex surfaces of general type, holds for symplectic 4-manifolds.

2.2. Lefschetz fibrations and stabilization by symplectic sums. One possible approach to the classification of symplectic 4-manifolds is via symplectic Lefschetz fibrations, as suggested by Donaldson. After blowing up a certain number of points, every compact integral symplectic 4-manifold can be realized as the total space of a fibration over $S^{2}$ whose fibers are compact Riemann surfaces, finitely many of which present a nodal singularity 9 . Conversely, the total space of such a Lefschetz fibration is a symplectic 4manifold 16. If one could classify symplectic Lefschetz fibrations, then an answer to Question 2.1 would follow. 
When the fiber genus is 0 or 1 , the classification of Lefschetz fibrations is a classical result; in particular, these fibrations are all holomorphic 22 . For genus 2, Siebert and Tian have proved holomorphicity under assumptions of irreducibility of the singular fibers and transitivity of the monodromy [26], but in general there are non-holomorphic examples [24, and the complete classification is not known. However, the situation simplifies if we "stabilize" by repeatedly performing fiber sums with a specific holomorphic fibration $f_{0}$ (the fibration obtained by blowing up a pencil of curves of bidegree $(2,3)$ in $\left.\mathbb{C P}^{1} \times \mathbb{C P}^{1}\right)$. Then we have the following result [2]:

Theorem 2.3. For any genus 2 symplectic Lefschetz fibration $f: X \rightarrow S^{2}$, there exists an integer $n_{0}$ such that, for all $n \geq n_{0}, f \# n f_{0}$ is isomorphic to a holomorphic fibration.

In fact, given two genus 2 symplectic Lefschetz fibrations $f, f^{\prime}$ with the same numbers of singular fibers of each type (irreducible, reducible with genus 1 components, reducible with components of genus 0 and 2), for all large $n$ the fiber sums $f \# n f_{0}$ and $f^{\prime} \# n f_{0}$ are isomorphic [2]. More generally, as a corollary of a recent result of Kharlamov and Kulikov about braid monodromy factorizations [19, a similar result holds for all Lefschetz fibrations with monodromy contained in the hyperelliptic mapping class group. This leads to the following questions relative to the classification of symplectic 4-manifolds up to stabilization by fiber sums:

Question 2.4. Does every symplectic Lefschetz fibration become isomorphic to a holomorphic fibration after repeatedly fiber summing with certain standard holomorphic fibrations?

Question 2.5. Let $X_{1}, X_{2}$ be two integral compact symplectic 4-manifolds with the same $\left(c_{1}^{2}, c_{2}, c_{1} \cdot[\omega],[\omega]^{2}\right)$. Do $X_{1}$ and $X_{2}$ become symplectomorphic after repeatedly performing symplectic sums with the same complex projective surfaces (chosen among a finite collection of model surfaces)?

2.3. Luttinger surgery. Many of the constructions used to obtain interesting examples of non-Kähler symplectic 4-manifolds, such as symplectic sum, link surgery, and symplectic rational blowdown, rely on the idea of cutting and pasting elementary building blocks. We focus here on the construction known as Luttinger surgery [21, which has been comparatively less studied but can be used to provide a unified description of numerous examples of exotic symplectic 4-manifolds.

Given an embedded Lagrangian torus $T$ in a symplectic 4-manifold $(X, \omega)$ and a homotopically non-trivial embedded loop $\gamma \subset T$, Luttinger surgery is an operation that consists in cutting out from $X$ a tubular neighborhood of $T$, foliated by parallel Lagrangian tori, and gluing it back in such a way that the new meridian loop differs from the old one by a twist along the loop $\gamma$ (while longitudes are not affected), yielding a new symplectic manifold $(\tilde{X}, \tilde{\omega})$. 
More precisely, identify a neighborhood of $T$ in $X$ with the neighborhood $T^{2} \times D^{2}(r)$ of the zero section in $\left(T^{*} T^{2}, d p_{1} \wedge d q_{1}+d p_{2} \wedge d q_{2}\right)$, in such a way that $\gamma$ is identified with the first factor in $T^{2}=S^{1} \times S^{1}$. Let $\theta$ be a smooth circle-valued function on the annulus $A=D^{2}(r) \backslash D^{2}\left(\frac{r}{2}\right)$ such that $\partial \theta / \partial p_{2}=0$, and representing the generator of $H^{1}(A)=\mathbb{Z}$ (i.e., the value of $\theta$ increases by $2 \pi$ as one goes around the origin). The diffeomorphism of $T^{2} \times A$ defined by

$$
\phi\left(q_{1}, q_{2}, p_{1}, p_{2}\right)=\left(q_{1}+\theta\left(p_{1}, p_{2}\right), q_{2}, p_{1}, p_{2}\right)
$$

preserves the symplectic form, and so the manifold

$$
\tilde{X}=\left(X \backslash T^{2} \times D^{2}\left(\frac{r}{2}\right)\right) \cup_{\phi}\left(T^{2} \times D^{2}(r)\right)
$$

inherits a natural symplectic structure. For more details see [21, 3].

By performing Luttinger surgery along suitably chosen Lagrangian tori, one can e.g. transform a product $T^{2} \times \Sigma$ into any surface bundle over $T^{2}$, or an untwisted fiber sum of Lefschetz fibrations into a twisted fiber sum. Fintushel and Stern's symplectic examples of knot surgery manifolds can also be obtained from complex surfaces by Luttinger surgery. Although there is no good reason to believe that the answer should be positive, the wide range of examples which reduce to this construction makes it interesting to ask the following question:

Question 2.6. Let $X_{1}, X_{2}$ be two integral compact symplectic 4-manifolds with the same $\left(c_{1}^{2}, c_{2}, c_{1} \cdot[\omega],[\omega]^{2}\right)$. Is it always possible to obtain $X_{2}$ from $X_{1}$ by a sequence of Luttinger surgeries?

In this question, as in Question 2.5 above, we do not require the fundamental groups of $X_{1}$ and $X_{2}$ to be isomorphic. This is because Luttinger surgery, like symplectic sum, can drastically modify the fundamental group. Also, let us mention that a positive answer to Question 2.6 essentially implies a positive answer to Question 2.5. as we shall see in $\S 4$.

The symplectic sum construction can be used to build minimal simply connected symplectic 4-manifolds with Chern numbers violating the Noether inequality, and hence not diffeomorphic to any complex surface (see e.g. Theorem 10.2.14 in [16]). Many of these manifolds are homeomorphic to (non-minimal) complex surfaces, but it is not clear at all whether it is possible to obtain them by Luttinger surgeries. Given the very explicit nature of the construction, these could be good test examples for Question 2.6]

\section{Isotopy QUeStions ABout SINGUlar Plane CURVES}

3.1. Symplectic branched covers. Let $X$ and $Y$ be compact oriented 4-manifolds, and assume that $Y$ carries a symplectic form $\omega_{Y}$.

Definition 3.1. A smooth map $f: X \rightarrow Y$ is a symplectic branched covering if given any point $p \in X$ there exist neighborhoods $U \ni p, V \ni f(p)$, and local coordinate charts $\phi: U \rightarrow \mathbb{C}^{2}$ (orientation-preserving) and $\psi: V \rightarrow \mathbb{C}^{2}$ 
(adapted to $\omega_{Y}$, i.e. such that $\omega_{Y}$ restricts positively to any complex line in $\left.\mathbb{C}^{2}\right)$, in which $f$ is given by one of:

(i) $(x, y) \mapsto(x, y)$ (local diffeomorphism),

(ii) $(x, y) \mapsto\left(x^{2}, y\right)$ (simple branching),

(iii) $(x, y) \mapsto\left(x^{3}-x y, y\right)$ (ordinary cusp).

These local models are the same as for the singularities of a generic holomorphic map from $\mathbb{C}^{2}$ to itself, except that the requirements on the local coordinate charts have been substantially weakened. The ramification curve $R=\{p \in X, \operatorname{det}(d f)=0\}$ is a smooth submanifold of $X$, and its image $D=f(R)$ is the branch curve, described in the local models by the equations $z_{1}=0$ for $(x, y) \mapsto\left(x^{2}, y\right)$ and $27 z_{1}^{2}=4 z_{2}^{3}$ for $(x, y) \mapsto\left(x^{3}-x y, y\right)$. It follows from the definition that $D$ is a singular symplectic curve in $Y$. Generically, its only singularities are transverse double points, which may occur with either the complex orientation or the opposite orientation, and complex cusps. We have the following result [1]:

Proposition 3.2. Given a symplectic branched covering $f: X \rightarrow Y$, the manifold $X$ inherits a natural symplectic structure $\omega_{X}$, canonical up to isotopy, in the cohomology class $\left[\omega_{X}\right]=f^{*}\left[\omega_{Y}\right]$.

The symplectic form $\omega_{X}$ is constructed by adding to $f^{*} \omega_{Y}$ a small multiple of an exact form $\alpha$ with the property that, at every point of $R$, the restriction of $\alpha$ to $\operatorname{Ker}(d f)$ is positive. Uniqueness up to isotopy follows from the convexity of the space of such exact 2-forms and Moser's theorem.

Conversely, we can realize every integral compact symplectic 4-manifold as a symplectic branched cover of $\mathbb{C P}^{2}$ [1]:

Theorem 3.3. Given an integral compact symplectic 4-manifold $\left(X^{4}, \omega\right)$ and an integer $k \gg 0$, there exists a symplectic branched covering $f_{k}: X \rightarrow$ $\mathbb{C P}^{2}$, canonical up to isotopy if $k$ is sufficiently large.

The maps $f_{k}$ are built from suitably chosen triples of sections of $L^{\otimes k}$, where $L \rightarrow X$ is a complex line bundle such that $c_{1}(L)=[\omega]$. In the complex case, $L$ is an ample line bundle, and a generic triple of holomorphic sections of $L^{\otimes k}$ determines a $\mathbb{C P}^{2}$-valued map $f_{k}: p \mapsto\left[s_{0}(p): s_{1}(p): s_{2}(p)\right]$. In the symplectic case the idea is similar, but requires more analysis; the proof relies on asymptotically holomorphic methods [1].

In any case, the natural symplectic structure induced on $X$ by the FubiniStudy Kähler form and $f_{k}$ (as given by Proposition [3.2) agrees with $\omega$ up to isotopy and scaling (multiplication by $k$ ).

Because for large $k$ the maps $f_{k}$ are canonical up to isotopy through symplectic branched covers, the topology of $f_{k}$ and of its branch curve $D_{k}$ can be used to define invariants of the symplectic manifold $(X, \omega)$. Although the only generic singularities of the plane curve $D_{k}$ are nodes (transverse double points) of either orientation and complex cusps, in a generic one-parameter family of branched covers pairs of nodes with opposite orientations may be 
cancelled or created. However, recalling that a node of $D_{k}$ corresponds to the occurrence of two simple branch points in a same fiber of $f_{k}$, the creation of a pair of nodes can only occcur in a manner compatible with the branched covering structure, i.e. involving disjoint sheets of the covering.

It is worth mentioning that, to this date, there is no evidence suggesting that negative nodes actually do occur in these high degree branch curves; our inability to rule our their presence might well be a shortcoming of the approximately holomorphic techniques, rather than an intrinsic feature of symplectic 4-manifolds. So we will occasionally consider the more conventional problem of understanding isotopy classes of curves presenting only positive nodes and cusps, although most of the discussion applies equally well to curves with negative nodes.

Assuming that the topology of the branch curve is understood, the structure of $f$ is determined by its monodromy morphism $\theta: \pi_{1}\left(\mathbb{C P}^{2}-D\right) \rightarrow S_{N}$, where $N$ is the degree of the covering $f$. Fixing a base point $p_{0} \in \mathbb{C P}^{2}-D$, the image by $\theta$ of a loop $\gamma$ in the complement of $D$ is the permutation of the fiber $f^{-1}\left(p_{0}\right)$ induced by the monodromy of $f$ along $\gamma$. (Since viewing this permutation as an element of $S_{N}$ depends on the choice of an identification between $f^{-1}\left(p_{0}\right)$ and $\{1, \ldots, N\}$, the morphism $\theta$ is only well-defined up to conjugation by an element of $S_{N}$.) By Proposition 3.2. the isotopy class of the branch curve $D$ and the monodromy morphism $\theta$ determine completely the symplectic 4-manifold $(X, \omega)$ up to symplectomorphism.

The image by $\theta$ of a geometric generator of $\pi_{1}\left(\mathbb{C P}^{2}-D\right)$, i.e. a loop $\gamma$ which bounds a small topological disc intersecting $D$ transversely once, is a transposition (because of the local model near a simple branch point). Since the image of $\theta$ is generated by transpositions and acts transitively on the fiber (assuming $X$ to be connected), $\theta$ is a surjective group homomorphism. Moreover, the smoothness of $X$ above the singular points of $D$ imposes certain compatibility conditions on $\theta$. Therefore, not every singular plane curve can be the branch curve of a smooth covering; in fact, the morphism $\theta$, if it exists, is often unique (up to conjugation in $S_{N}$ ). In the case of algebraic curves, this uniqueness property, which holds except for a finite list of well-known counterexamples, is known as Chisini's conjecture, and was essentially proved by Kulikov a few years go [20].

The upshot of the above discussion is that, in order to understand symplectic 4-manifolds, it is in principle enough to understand singular plane curves. Moreover, if the branch curve of a symplectic covering $f: X \rightarrow \mathbb{C P}^{2}$ happens to be a complex curve, then the integrable complex structure of $\mathbb{C P}^{2}$ can be lifted to an integrable complex structure on $X$, compatible with the symplectic structure; this implies that $X$ is a complex projective surface. So, considering the branched coverings constructed in Theorem 3.3. we have: 
Corollary 3.4. For $k \gg 0$ the branch curve $D_{k} \subset \mathbb{C P}^{2}$ is isotopic to a complex curve (up to node cancellations) if and only if $X$ is a complex projective surface.

This motivates the study of the symplectic isotopy problem for singular curves in $\mathbb{C P}^{2}$ (or more generally in other complex surfaces - especially rational ruled surfaces, i.e. $\mathbb{C P}^{1}$-bundles over $\mathbb{C P}^{1}$ ).

3.2. The symplectic isotopy problem. The symplectic isotopy problem asks under which circumstances (assumptions on degree, singularities, ...) it is true that any symplectic curve is isotopic to a complex curve (by isotopy, we mean a continuous one-parameter family of symplectic curves with the same singularities). More generally, the goal is to understand isotopy classes of symplectic curves with given singularities in a given homology class. For example, considering only plane curves with positive nodes and cusps, one may ask the following:

Question 3.5. Given integers $(d, \nu, \kappa)$, can one classify all symplectic curves of degree $d$ in $\mathbb{C P}^{2}$ with $\nu$ nodes and $\kappa$ cusps, up to symplectic isotopy?

If $D$ is the branch curve of an $N$-fold symplectic covering, then the Chern classes of the symplectic manifold $(X, \omega)$ (with the symplectic structure given by Proposition [3.2) are related to the degree $d$ of $D$, its genus $g=$ $\frac{1}{2}(d-1)(d-2)-\kappa-\nu$, and its number of cusps via the formulas:

$[\omega]^{2}=N, \quad c_{1} \cdot[\omega]=3 N-d, \quad c_{1}^{2}=g-1-\frac{9}{2} d+9 N, \quad c_{2}=2 g-2+3 N-\kappa$.

In particular, integrality constraints on the Euler-Poincaré characteristic $\chi=c_{2}$ and signature $\sigma=\frac{1}{3}\left(c_{1}^{2}-2 c_{2}\right)$ of $X$ imply that the degree $d$ must be even, and that the number of cusps $\kappa$ must be a multiple of 3 . The geography problem for symplectic 4-manifolds translates into a geography problem for symplectic branch curves: for example, the Bogomolov-Miyaoka-Yau inequality $c_{1}^{2} \leq 3 c_{2}$ translates into the inequality

$$
\kappa \leq \frac{5}{3}(g-1)+\frac{3}{2} d .
$$

There are plane curves which violate this inequality, even in the algebraic world: e.g. the branch curves of generic projections of irrational ruled surfaces $\Sigma \times \mathbb{C P}^{1}$, where $\Sigma$ is a curve of genus $\geq 2$. However, the open question is whether one can find branch curves which violate this inequality and for which the branched covering has $c_{1}^{2} \geq 0$. By the above remarks, these cannot be isotopic to any complex curve.

The symplectic isotopy problem is understood in various simple situations, where it can be shown that every symplectic curve is isotopic to a complex curve. The first results were obtained by Gromov [17, who used pseudoholomorphic curves to prove that every smooth symplectic curve of degree 1 or 2 in $\mathbb{C P}^{2}$ is isotopic to a complex curve. The idea of the argument is to equip $\mathbb{C P}^{2}$ with an almost-complex structure $J=J_{1}$ such that the given curve $C$ 
is $J$-holomorphic, and consider a smooth family of almost-complex structures $\left(J_{t}\right)_{t \in[0,1]}$ interpolating between $J$ and the standard complex structure $J_{0}$. By studying the deformation problem for pseudoholomorphic curves, one can prove the existence of a smooth family of $J_{t}$-holomorphic curves $C_{t}$ realizing an isotopy between $C=C_{1}$ and an honest holomorphic curve $C_{0}$.

Successive improvements of this result have been obtained by Sikorav (for smooth curves of degree $\leq 3$ ), Shevchishin (degree $\leq 6$ ), and more recently Siebert and Tian [26]:

Theorem 3.6 (Siebert-Tian). Every smooth symplectic curve of degree $\leq 17$ in $\mathbb{C P}^{2}$ is isotopic to a complex curve.

A similar result has also been obtained for smooth curves in $\mathbb{C P}^{1}$-bundles over $\mathbb{C P}^{1}$ (assuming $[C] \cdot[$ fiber] $\leq 7$ ) [26]. It is expected that the isotopy property remains true for smooth plane curves of arbitrarily large degree; this would provide an answer to Question 3.5 in the case $\nu=\kappa=0$ (recall that all smooth complex curves of a given degree are mutually isotopic).

The isotopy property is also known to hold in some simple cases for curves with nodes and cusps in $\mathbb{C P}^{2}$ and $\mathbb{C P}^{1}$-bundles over $\mathbb{C P}^{1}$, as illustrated by the results obtained by Barraud, Shevchishin, and Francisco. For example, we have the following results 25,12 :

Theorem 3.7 (Shevchishin). Any two irreducible nodal symplectic curves in $\mathbb{C P}^{2}$ of the same degree and the same genus $g \leq 4$ are symplectically isotopic.

Theorem 3.8 (Francisco). Let $C$ be an irreducible symplectic curve of degree $d$ and genus 0 with $\kappa$ cusps and $\nu$ nodes in $\mathbb{C P}^{2}$, and assume that $\kappa<d$. Then $C$ is isotopic to a complex curve.

In general, we cannot expect the classification to be so simple, and there are plenty of examples of symplectic curves which are not isotopic to any complex curve. Perhaps the most widely known such examples are due to Fintushel and Stern [11, who showed that elliptic surfaces contain infinite families of pairwise non-isotopic smooth symplectic curves representing a same homology class. Similar results have also been obtained by Smith, Etgü and Park, and Vidussi. However, if we consider singular curves with cusp singularities, then these non-isotopy phenomena already arise in $\mathbb{C P}^{2}$. In a non-explicit manner, it is clear that this must be the case, from Corollary [3.4. however to this date the branch curves given by Theorem 3.3 for $k \gg 0$ have not been computed explicitly for any non-complex examples. More explicitly, the following result is due to Moishezon [23] (see also [3]):

Theorem 3.9 (Moishezon). For all $p \geq 2$, there exist infinitely many pairwise non-isotopic singular symplectic curves of degree $d=9 p(p-1)$ in $\mathbb{C P}^{2}$ with $\kappa=27(p-1)(4 p-5)$ cusps and $\nu=\frac{27}{2}(p-1)(p-2)\left(3 p^{2}+3 p-8\right)$ nodes, not isotopic to any complex curve. 
Moishezon's approach is purely algebraic (using braid monodromy factorizations), and yields curves that are distinguished by the fundamental groups of their complements 23. However a simpler geometric description of his construction can be given in terms of braiding constructions [3] cf. §3.4.

Questions 2.1 and 3.5 are closely related to each other, via Proposition 3.2 and Theorem 3.3. Let us restrict ourselves to those plane curves which admit a compatible symmetric group valued monodromy morphism, and assume that Chisini's conjecture about the uniqueness of this morphism (excluding a specific degree 6 curve) extends to the symplectic case. Then integral compact symplectic 4-manifolds (up to scaling of the symplectic form) are in one-to-one correspondence with isotopy classes of singular symplectic plane branch curves up to an equivalence relation which takes into account: (1) the possibility of creating and cancelling pairs of nodes, and (2) the dependence of the branch curve $D_{k}$ on the parameter $k$ in Theorem 3.3. This latter dependence, while complicated and not quite understood in general, is nonetheless within reach: see [6] for a description of the relation between $D_{k}$ and $D_{2 k}$.

If one allows creations and cancellations of pairs of nodes, then the classification problem becomes different, even considering only curves with positive nodes and cusps. Indeed, it may happen that two non-isotopic curves can be deformed into each other if one is allowed to "push" the curve through itself, creating or cancelling pairs of double points in the process (such a deformation is called a regular homotopy). In fact, in this case the classification becomes excessively simple, as shown by the following result [7]:

Theorem 3.10 (A.-Kulikov-Shevchishin). Any two irreducible symplectic curves with positive nodes and cusps in $\mathbb{C P}^{2}$, of the same degree and with the same numbers of nodes and cusps, are regular homotopic to each other.

What this means is that, when considering symplectic branch curves given by Theorem 3.3. it is important to restrict oneself to admissible regular homotopies, i.e. regular homotopies which are compatible with the symmetric group valued monodromy morphism $\theta$. When pushing the branch curve $D$ through itself, the two branches that are made to intersect each give rise to a geometric generator of $\pi_{1}\left(\mathbb{C P}^{2}-D\right)$. The requirement for admissibility of a node creation operation is that the images by $\theta$ of these two geometric generators should be transpositions acting on disjoint pairs of elements (i.e., the branching phenomena above the two intersecting branches of $D$ should occur in different sheets of the covering). Thus the version of the isotopy problem which naturally comes out of Theorem 3.3 is the following:

Question 3.11. Given integers $(d, \nu, \kappa, N)$, can one classify all pairs $(D, \theta)$ where $D$ is a symplectic curve of degree $d$ in $\mathbb{C P}^{2}$ with $\nu_{+}$positive nodes, $\nu_{-}$ negative nodes and $\kappa$ complex cusps, $\nu_{+}-\nu_{-}=\nu$, and $\theta: \pi_{1}\left(\mathbb{C P}^{2}-D\right) \rightarrow$ $S_{N}$ is a compatible monodromy morphism, up to admissible regular homotopies? 
3.3. Hurwitz curves and stabilization. In order to state the analogue of Question 2.4 for branch curves, we need to introduce a slightly more restrictive category of curves, known at Hurwitz curves. Roughly speaking, a Hurwitz curve in a ruled surface is a curve which behaves like a generic complex curve with respect to the ruling. In the case of $\mathbb{C P}^{2}$, we consider the projection $\pi: \mathbb{C P}^{2}-\{(0: 0: 1)\} \rightarrow \mathbb{C P}^{1}$ given by $(x: y: z) \mapsto(x: y)$, and we make the following definition:

Definition 3.12. A curve $D \subset \mathbb{C P}^{2}$ (not passing through $(0: 0: 1)$ ) is a Hurwitz curve if $D$ is positively transverse to the fibers of $\pi$ at all but finitely many points, where $D$ is smooth and non-degenerately tangent to the fibers.

Hurwitz curves in $\mathbb{C P}^{1}$-bundles over $\mathbb{C P}^{1}$ can be defined similarly, considering the projection to $\mathbb{C P}^{1}$ given by the bundle structure.

It is easy to see that any Hurwitz curve in $\mathbb{C P}^{2}$ can be made symplectic by an isotopy through Hurwitz curves: namely, the image of any Hurwitz curve by the rescaling map $(x: y: z) \mapsto(x: y: \lambda z)$ is a Hurwitz curve, and symplectic for $|\lambda| \ll 1$. Moreover, Theorem 3.3 can be improved to ensure that the branch curves $D_{k} \subset \mathbb{C P}^{2}$ are Hurwitz curves [5]. So, the discussion in $\S \S 3.1-3.2$ carries over to the world of Hurwitz curves without modification.

After blowing up $\mathbb{C P}^{2}$ at $(0: 0: 1)$, we obtain the Hirzebruch surface $\mathbb{F}_{1}$ (recall that $\left.\mathbb{F}_{n}=\mathbb{P}\left(\mathcal{O}_{\mathbb{P}^{1}} \oplus \mathcal{O}_{\mathbb{P}^{1}}(n)\right)\right)$, and any Hurwitz curve in $\mathbb{C P}^{2}$ becomes a Hurwitz curve in $\mathbb{F}_{1}$, disjoint from the exceptional section. The advantage of considering Hurwitz curves in Hirzebruch surfaces rather than $\mathbb{C P}^{2}$ is that we can now introduce an operation of stabilization by pairwise fiber sum. Namely, consider two Hurwitz curves $D_{1} \subset \mathbb{F}_{n_{1}}, D_{2} \subset \mathbb{F}_{n_{2}}$, of the same degree $d$ relatively to the projection, i.e. such that $\left[D_{1}\right] \cdot[F]=\left[D_{2}\right] \cdot[F]=d$, where $F$ is the fiber of the ruling. Then, up to an isotopy among Hurwitz curves, we can assume that the intersections of $D_{1}$ and $D_{2}$ with fixed fibers of the rulings coincide, and we can smooth the normal crossing configuration $\left(\mathbb{F}_{n_{1}}, D_{1}\right) \cup_{\text {fiber=fiber }}\left(\mathbb{F}_{n_{2}}, D_{2}\right)$ into a pair $\left(\mathbb{F}_{n}, D\right)$, where $D$ is a Hurwitz curve in $\mathbb{F}_{n}$, and $n=n_{1}+n_{2}$.

If a Hurwitz curve in $\mathbb{F}_{n}$ is a branch curve, then the ruling on $\mathbb{F}_{n}$ lifts to a symplectic Lefschetz fibration on the branched cover. Assuming that the symmetric group valued morphisms are compatible (i.e. have the same restrictions to given fibers), the fiber sum operation on the branch curves then corresponds to a fiber sum operation on the covers. Hence, the analogue of Questions 2.4 and 2.5 asks whether stabilization by fiber summing can be used to simplify the classification of Hurwitz branch curves:

Question 3.13. Let $D_{1}, D_{2}$ be two Hurwitz curves in $\mathbb{F}_{n}$, representing the same homology class and with the same numbers of cusps and nodes. Assume that two compatible monodromy morphisms $\theta_{i}: \pi_{1}\left(\mathbb{F}_{n}-D_{i}\right) \rightarrow S_{N}$ are given $(i \in\{1,2\})$, and that there is a fiber $F \subset \mathbb{F}_{n}$ such that $F \cap D_{1}=F \cap D_{2}$ and $\theta_{1 \mid F-D_{1}}=\theta_{2 \mid F-D_{2}}$. Is there a complex curve $C \subset \mathbb{F}_{n^{\prime}}$, compatible with the 
given monodromy morphisms, such that the fiber sums $D_{1} \# C$ and $D_{2} \# C$ are isotopic to each other as Hurwitz curves?

To remain closer to the formulation of the questions in $\S 2$, one can instead require the complex curve to be chosen among a finite list of standard models (depending on the given monodromy morphisms $\theta_{i}$ ), but allow several successive fiber sum operations. It is also interesting to ask whether the final result of the fiber sum operations can always be assumed to be isotopic to a complex curve.

Requiring compatibility with the given monodromy morphisms places restrictions on the choice of the curve $C$, and makes the question more difficult. Without this constraint the answer is known, and follows directly from a recent result of Kharlamov and Kulikov about braid monodromy factorizations 19:

Theorem 3.14 (Kharlamov-Kulikov). Let $D_{1}, D_{2}$ be two Hurwitz curves in $\mathbb{F}_{n}$, representing the same homology class and with the same numbers of cusps and nodes. Then there exists a smooth complex curve $C$ in $\mathbb{F}_{0}=$ $\mathbb{C P}^{1} \times \mathbb{C P}^{1}$ such that the fiber sums $D_{1} \# C$ and $D_{2} \# C$ are isotopic.

In this result, $C$ is in fact a smooth curve of bidegree $(a, b)$, where $a=$ $[F] \cdot\left[D_{i}\right]$, and $b \gg 0$ is chosen very large. Such a curve may be obtained by smoothing a configuration consisting of $a$ sections $\mathbb{C P}^{1} \times\{p t\}$ and $b$ fibers $\{p t\} \times \mathbb{C P}^{1}$. Hence, in this case the fiber sum operation is equivalent to considering the union of $D_{i}$ with $b$ fibers of the ruling, and smoothing the intersections; a more geometric formulation of Theorem 3.14 is therefore:

Theorem 3.15. Let $D_{1}, D_{2}$ be two Hurwitz curves in $\mathbb{F}_{n}$, representing the same homology class and with the same numbers of cusps and nodes. Let $D_{i}^{\prime}$ $(i \in\{1,2\})$ be the curve obtained by adding to $D_{i}$ a union of $b$ generic fibers of the ruling, intersecting $D_{i}$ transversely at smooth points, and smoothing out all the resulting intersections. Then for all large enough values of $b$ the Hurwitz curves $D_{1}^{\prime}$ and $D_{2}^{\prime}$ are isotopic.

This construction gives an answer to Question 3.13 in the case of smooth curves (and coverings of degree $N=2$ ); it is unclear whether the argument in [19] can be modified to produce complex curves compatible with branched coverings of degree $N \geq 3$.

3.4. Braiding along Lagrangian annuli. Let $D$ be a symplectic curve in a symplectic 4-manifold $Y$ (e.g. $Y=\mathbb{C P}^{2}$ ), possibly with singularities. It is often the case that we can find an embedded Lagrangian annulus $A \subset Y \backslash D$, with boundary contained in the smooth part of $D$. (This happens for example when a portion of $D$ consists of two cylinders which run parallel to each other; then we can find a Lagrangian annulus joining them).

In this situation, one can twist the curve $D$ along the annulus $A$, to obtain a new symplectic curve $\tilde{D}$ which coincides with $D$ away from $A[3$. Namely, we can identify a neighborhood of $A$ with the product $S^{1} \times(-1,1) \times D^{2}$, 
in such a way that $A=S^{1} \times\{0\} \times\left[-\frac{1}{2}, \frac{1}{2}\right]$ and a neighborhood of $\partial A$ in $D$ is $S^{1} \times(-1,1) \times\left\{ \pm \frac{1}{2}\right\}$. (If we deform $D$ suitably, then we may assume that the symplectic structure is the product one, but this is not necessary). Then the curve $\tilde{D}$ is obtained from $D$ by replacing $S^{1} \times(-1,1) \times\left\{ \pm \frac{1}{2}\right\}$ by $S^{1} \times \tilde{\Gamma}$, where $\tilde{\Gamma}=\left\{\left(t, \pm \frac{1}{2} \exp (i \pi \chi(t))\right), t \in(-1,1)\right\} \subset(-1,1) \times D^{2}$ and $\chi$ is a smooth function which equals 0 near -1 and 1 near 1 . This construction is called "braiding" because, forgetting the $S^{1}$ factor, it replaces the trivial braid $(-1,1) \times\left\{ \pm \frac{1}{2}\right\}$ with the half-twist $\tilde{\Gamma}$.

Assume now that $D$ is the branch curve of an $N$-fold symplectic covering $f: X \rightarrow Y$. Assume moreover that $f$ is ramified in the same manner above the two boundary components of $A$, i.e. that two of the $N$ lifts of $A$ have boundary contained in the ramification curve $R$; then these two lifts together form an embedded Lagrangian torus $T \subset X$, and we have the following result $[3]$ :

Proposition 3.16 (A.-Donaldson-Katzarkov). The symplectic 4-manifold $\tilde{X}$ obtained from $X$ by Luttinger surgery along the torus $T$ is the total space of a natural symplectic branched covering $\tilde{f}: \tilde{X} \rightarrow Y$, whose branch curve $\tilde{D}$ is the curve obtained from $D$ by braiding along the annulus $A$.

Hence, the natural analogue of Question 2.6 for singular plane curves is:

Question 3.17. Let $D_{1}, D_{2}$ be two symplectic curves with positive nodes and cusps in $\mathbb{C P}^{2}$, of the same degree and with the same numbers of nodes and cusps. Is it always possible to obtain $D_{2}$ from $D_{1}$ by a sequence of braiding operations along Lagrangian annuli?

As before, there is no good reason to believe that the answer should be positive, except that most known examples of non-isotopic symplectic curves seem to reduce to this construction. This is e.g. the case for the FintushelStern examples of non-isotopic smooth symplectic curves in elliptic surfaces [1], which are obtained by braiding a disconnected union of elliptic fibers, and for Moishezon's examples of singular plane curves [23, 3], which are obtained by braiding the branch curve of the projection of a complex surface of general type.

\section{Questions ABOUT BRAID MONODROMY FACTORIZATIONS}

4.1. The braid monodromy of a plane curve. One of the main tools to study algebraic plane curves is the notion of braid monodromy, which has been used extensively by Moishezon and Teicher (among others) since the early 1980s in order to study the branch curves of generic projections of complex projective surfaces (see 30] for a detailed overview). Braid monodromy techniques apply equally well to the more general case of Hurwitz curves in $\mathbb{C P}^{2}$ or more generally in rational ruled surfaces (see Definition 3.12).

Given a Hurwitz curve $D$ in $\mathbb{C P}^{2}$, the projection $\pi: \mathbb{C P}^{2}-\{(0: 0: 1)\} \rightarrow$ $\mathbb{C P}^{1}$ makes $D$ a singular branched cover of $\mathbb{C P}^{1}$, of degree $d=\operatorname{deg} D$. Each 
fiber of $\pi$ is a complex line $\ell \simeq \mathbb{C} \subset \mathbb{C P}^{2}$, and if $\ell$ does not pass through any of the singular points of $D$ nor any of its vertical tangencies, then $\ell \cap D$ consists of $d$ distinct points. We can trivialize the fibration $\pi$ over an affine subset $\mathbb{C} \subset \mathbb{C P}^{1}$, and define the braid monodromy morphism

$$
\rho: \pi_{1}\left(\mathbb{C}-\operatorname{crit}\left(\pi_{\mid D}\right)\right) \rightarrow B_{d}
$$

Here $B_{d}$ is the Artin braid group on $d$ strings (the fundamental group of the configuration space $\mathcal{X}_{d}$ of $d$ distinct points in $\mathbb{C}$ ), and for any loop $\gamma$ the braid $\rho(\gamma)$ describes the motion of the $d$ points of $\ell \cap D$ inside the fibers of $\pi$ as one moves along the loop $\gamma$.

Equivalently, choosing an ordered system of arcs generating the free group $\pi_{1}\left(\mathbb{C}-\operatorname{crit}\left(\pi_{\mid D}\right)\right)$, one can express the braid monodromy of $D$ by a factorization

$$
\Delta^{2}=\prod_{i} \rho_{i}
$$

of the central element $\Delta^{2}$ (representing a full rotation by $2 \pi$ ) in $B_{d}$, where each factor $\rho_{i}$ is the monodromy around one of the special points (cusps, nodes, tangencies) of $D$.

The monodromy around a tangency point is a half-twist exchanging two strands, i.e. an element conjugated to one of the standard generators of $B_{d}$; the monodromy around a positive (resp. negative) node is the square (resp. the inverse of the square) of a half-twist; and the monodromy around a cusp is the cube of a half-twist. Hence, we are interested in factorizations of $\Delta^{2}$ into products of powers of half-twists.

A same Hurwitz curve can be described by different factorizations of $\Delta^{2}$ in $B_{d}$ : switching to a different ordered system of generators of $\pi_{1}\left(\mathbb{C}-\operatorname{crit}\left(\pi_{\mid D}\right)\right)$ affects the collection of factors $\left\langle\rho_{1}, \ldots, \rho_{r}\right\rangle$ by a sequence of Hurwitz moves, i.e. operations of the form

$$
\left\langle\rho_{1}, \cdots, \rho_{i}, \rho_{i+1}, \cdots, \rho_{r}\right\rangle \longleftrightarrow\left\langle\rho_{1}, \cdots,\left(\rho_{i} \rho_{i+1} \rho_{i}^{-1}\right), \rho_{i}, \cdots, \rho_{r}\right\rangle ;
$$

and changing the trivialization of the reference fiber $(\ell, \ell \cap D)$ of $\pi$ (i.e. its identification with the base point in $\mathcal{X}_{d}$ ) affects braid monodromy by a global conjugation

$$
\left\langle\rho_{1}, \cdots, \rho_{r}\right\rangle \longleftrightarrow\left\langle b^{-1} \rho_{1} b, \cdots, b^{-1} \rho_{r} b\right\rangle .
$$

For Hurwitz curves whose only singularities are cusps and nodes (of either orientation), the braid monodromy factorization determines the isotopy type completely (see for example [19]). Hence, determining whether two given Hurwitz curves are isotopic is equivalent to determining whether two given factorizations of $\Delta^{2}$ coincide up to Hurwitz moves and global conjugation. In this language the isotopy problem for Hurwitz curves in $\mathbb{C P}^{2}$ becomes:

Question 4.1. Given integers $(d, \nu, \kappa)$, can one classify all factorizations of the central element $\Delta^{2}$ of $B_{d}$ into a product of $\tau=d(d-1)-2 \nu-3 \kappa$ half-twists, $\nu$ squares of half-twists, and $\kappa$ cubes of half-twists, up to Hurwitz moves and global conjugation? 
If our goal is to consider only branch curves of symplectic coverings (rather than arbritrary plane Hurwitz curves), then we need to look specifically for factorizations in which the factors belong to the liftable braid group, i.e. the subgroup of $B_{d}$ consisting of all braids compatible with given branched covering data.

More precisely, assume that a Hurwitz curve $D$ is the branch curve of a symplectic branched covering $f: X \rightarrow \mathbb{C P}^{2}$. The fibers of $\pi$ form a pencil of lines on $\mathbb{C P}^{2}$, whose preimages by $f$ equip $X$ with a structure of symplectic Lefschetz pencil. By restricting the monodromy of the covering to a fiber $\ell$ of $\pi$, we obtain a symmetric group valued morphism

$$
\theta: \pi_{1}(\ell-(\ell \cap D)) \rightarrow S_{N}
$$

which describes how to realize a fiber of the Lefschetz pencil as a branched covering of a fiber of $\pi$. The braid group acts on $\pi_{1}(\ell-(\ell \cap D))$ by automorphisms; call $b_{*}$ the automorphism induced by the braid $b$. Then the liftable braid group is

$$
L B_{d}(\theta)=\left\{b \in B_{d}, \theta \circ b_{*}=\theta\right\} .
$$

Equivalently, recall that $B_{d}$ is the fundamental group of the space $\mathcal{X}_{d}$ of configurations of $d$ distinct points in $\mathbb{C}$, and consider the configuration space $\tilde{\mathcal{X}}_{d}$ whose elements are pairs $(\Pi, \sigma)$, where $\Pi$ is a set of $d$ distinct points in $\mathbb{C}$, and $\sigma$ is a surjective group homomorphism from $\pi_{1}(\mathbb{C}-\Pi)$ to $S_{N}$ mapping generators to transpositions. The projection $(\Pi, \sigma) \mapsto \Pi$ is a finite covering, and taking $\tilde{*}=(\ell \cap D, \theta)$ as base point we have $L B_{d}(\theta)=\pi_{1}\left(\tilde{\mathcal{X}}_{d}, \tilde{*}\right)$.

4.2. Stabilization and partial conjugation. The main feature which makes braid groups algorithmically manageable is the Garside property. Namely, if we consider the semigroup of positive braids $B_{d}^{+}$, defined by the same generators $\left(\sigma_{i}\right)_{1 \leq i \leq d-1}$ and relations $\left(\sigma_{i} \sigma_{i+1} \sigma_{i}=\sigma_{i+1} \sigma_{i} \sigma_{i+1} \quad \forall i\right.$ and $\left.\sigma_{i} \sigma_{j}=\sigma_{j} \sigma_{i} \forall|i-j| \geq 2\right)$ as $B_{d}$ but without allowing inverses of the generators, then we have the following property [13]:

Theorem 4.2 (Garside). The natural homomorphism $i: B_{d}^{+} \rightarrow B_{d}$ is an embedding.

In other terms, if two positive words in the generators of the braid group represent the same braid, then they can be transformed into each other by repeatedly using the defining relations, without ever introducing the inverses of the generators. Garside's other fundamental observation is that for any $b \in B_{d}$ there exists an integer $k$ and a positive braid $\beta \in B_{d}^{+}$such that $\Delta^{2 k} b=i(\beta)$ [13. These properties make it possible to obtain solutions to the word and conjugacy problems (see also [8] for a more modern approach); they also yield a stable classification of braid group factorizations [19].

Namely, let $\mathcal{F}_{0}$ be the standard factorization $\Delta^{2}=\left(\sigma_{1} \cdot \ldots \cdot \sigma_{d-1}\right)^{d}$ in $B_{d}$, and say that two factorizations $\mathcal{F}=\left(\rho_{1} \cdot \ldots \cdot \rho_{r}\right), \mathcal{F}^{\prime}=\left(\rho_{1}^{\prime} \cdot \ldots \cdot \rho_{r}^{\prime}\right)$ have the same number of factors of each type if they have the same number of 
factors $r$ and there is a permutation $\sigma \in S_{r}$ such that $\rho_{i}$ is conjugated to $\rho_{\sigma(i)}^{\prime}$ for all $i=1, \ldots, r$. Then the following result holds [19]:

Theorem 4.3 (Kharlamov-Kulikov). Let $\mathcal{F}$ and $\mathcal{F}^{\prime}$ be two factorizations of the same element in $B_{d}$, with the same numbers of factors of each type. Then there exists an integer $n$ such that the factorizations $\mathcal{F} \cdot\left(\mathcal{F}_{0}\right)^{n}$ and $\mathcal{F}^{\prime} \cdot\left(\mathcal{F}_{0}\right)^{n}$ are equivalent under Hurwitz moves.

(Here $\mathcal{F} \cdot\left(\mathcal{F}_{0}\right)^{n}$ is the factorization consisting of the factors of $\mathcal{F}$ followed by those of $\mathcal{F}_{0}$ repeated $n$ times).

Theorem 3.14 follows from this result by specifically considering factorizations of $\Delta^{2 n}$ whose factors are powers of half-twists and observing that $\mathcal{F}_{0}$ is the braid monodromy factorization of a smooth algebraic plane curve. However, considering that the factors in $\mathcal{F}_{0}$ generate the entire braid group, of which $L B_{d}(\theta)$ is a proper subgroup as soon as the degree $N$ of the covering is at least 3 , one is prompted to ask the following question:

Question 4.4. Given a symmetric group valued morphism $\theta$, does a statement similar to Theorem 4.3 hold for factorizations in $L B_{d}(\theta)$ ?

Assuming that the factorization in $L B_{d}(\theta)$ playing the role of the standard factorization $\mathcal{F}_{0}$ in this statement can be realized as the braid monodromy of an algebraic curve, a positive answer to this question would imply positive answers to Questions 2.5 and 3.13

Finally, the last question we will consider is that of partial conjugation of braid factorizations. Namely, given a factorization $\mathcal{F}$ with factors $\rho_{1}, \ldots, \rho_{r}$, integers $1 \leq p<q \leq r$, and a braid $b$ such that $\prod_{p \leq i \leq q} \rho_{i}$ commutes with $b$, we can form a new factorization $\mathcal{F}^{\prime}$, with factors $\rho_{1}, \ldots, \rho_{p-1},\left(b^{-1} \rho_{p} b\right), \ldots$, $\left(b^{-1} \rho_{q} b\right), \rho_{q+1}, \ldots, \rho_{r}$ : the partial conjugate of $\mathcal{F}$ by $b$.

Lemma 4.5. If the element $b$ belongs to the subgroup of $B_{d}$ generated by $\rho_{p}, \ldots, \rho_{q}$, and if $\prod_{p \leq i \leq q} \rho_{i}$ is central in this subgroup, then $\mathcal{F}^{\prime}$ is equivalent to $\mathcal{F}$ under Hurwitz moves.

The proof is easy, and relies on the same trick as in Lemma 6 of [2]. On the other hand, if $b$ does not belong to the subgroup generated by the factors of $\mathcal{F}$, then we can get interesting examples of inequivalent factorizations; this is e.g. how Moishezon's examples [23] are constructed.

Question 4.6. Are any two factorizations of the same element in $B_{d}$ (resp. $L B_{d}(\theta)$ ), with the same numbers of factors of each type, equivalent under Hurwitz moves and partial conjugations by elements of $B_{d}\left(\right.$ resp. $\left.L B_{d}(\theta)\right)$ ?

A positive answer to this question (for factorizations in $L B_{d}(\theta)$ ) would imply that Questions 2.6 and 3.17also admit positive answers. In fact, if one specifically considers factorizations of $\Delta^{2}$ into a product of powers of halftwists in $L B_{d}(\theta)$, then Questions [2.6] and 4.6] are almost equivalent. This is because, given an arbitrary Lagrangian torus $T$ in a symplectic 4-manifold, 
one can build a symplectic Lefschetz pencil for which $T$ fibers above an embedded loop $\delta$ in $\mathbb{C P}^{1}$ and intersects each fiber above $\delta$ in a simple closed loop $\gamma$. Luttinger surgery along $T$ then amounts to a partial conjugation of the monodromy of the pencil by the Dehn twist about $\gamma$, and considering branched coverings of $\mathbb{C P}^{2}$ instead of Lefschetz pencils it should also amount to a partial conjugation of the braid monodromy of the branch curve.

Moreover, a positive answer to Question [4.6 also implies a positive answer to Question 4.4 (and hence to Questions 2.5 and 3.13), at least provided that there exists an algebraic plane branch curve whose braid monodromy generates the liftable braid subgroup $L B_{d}(\theta)$. The existence of such a factorization $\mathcal{F}_{0, \theta}$ is rather likely, and examples should be relatively easy to find, although the question has not been studied. Assuming that this is the case, given two factorizations $\mathcal{F}_{1}, \mathcal{F}_{2}$ in $L B_{d}(\theta)$ with the same numbers of factors of each type, the factors in $\mathcal{F}_{1} \cdot \mathcal{F}_{0, \theta}$ and $\mathcal{F}_{2} \cdot \mathcal{F}_{0, \theta}$ generate $L B_{d}(\theta)$, and hence, by Lemma 4.5. any partial conjugation operation performed on $\mathcal{F}_{1} \cdot \mathcal{F}_{0, \theta}$ is equivalent to a sequence of Hurwitz moves. So, if $\mathcal{F}_{1} \cdot \mathcal{F}_{0, \theta}$ and $\mathcal{F}_{2} \cdot \mathcal{F}_{0, \theta}$ are equivalent under Hurwitz moves and partial conjugations then they are equivalent under Hurwitz moves only.

Note added in proof: Questions 2.4 and 2.5 have now essentially been solved. The reader is referred to: D. Auroux, A stable classification of Lefschetz fibrations, Geom. Topol. 9 (2005), 203-217.

\section{REFERENCES}

[1] D. Auroux, Symplectic 4-manifolds as branched coverings of $\mathbb{C P}^{2}$, Invent. Math. 139 (2000), 551-602.

[2] D. Auroux, Fiber sums of genus 2 Lefschetz fibrations, Turkish J. Math. 27 (2003), 1-10 (math.GT/0204285).

[3] D. Auroux, S. K. Donaldson, L. Katzarkov, Luttinger surgery along Lagrangian tori and non-isotopy for singular symplectic plane curves, Math. Ann. 326 (2003), 185203.

[4] D. Auroux, S. K. Donaldson, L. Katzarkov, Singular Lefschetz pencils, preprint.

[5] D. Auroux, L. Katzarkov, Branched coverings of $\mathbb{C P}^{2}$ and invariants of symplectic 4-manifolds, Invent. Math. 142 (2000), 631-673.

[6] D. Auroux, L. Katzarkov, The degree doubling formula for braid monodromies and Lefschetz pencils, preprint.

[7] D. Auroux, V. S. Kulikov, V. V. Shevchishin, Regular homotopy of Hurwitz curves, Izv. Math. 68 (2004), 521-542 (math.SG/0401172).

[8] J. Birman, K. H. Ko, S. J. Lee, A new approach to the word and conjugacy problems in the braid groups, Adv. Math. 139 (1998), 322-353.

[9] S. K. Donaldson, Lefschetz pencils on symplectic manifolds, J. Differential Geom. 53 (1999), 205-236.

[10] R. Fintushel, R. Stern, Knots, links, and 4-manifolds, Invent. Math. 134 (1998), 363-400.

[11] R. Fintushel, R. Stern, Symplectic surfaces in a fixed homology class, J. Differential Geom. 52 (1999), 203-222.

[12] S. Francisco, Symplectic isotopy problem for cusp curves, preprint, in preparation.

[13] F. A. Garside, The braid group and other groups, Quart. J. Math. Oxford 20 (1969), $235-254$. 
[14] D. T. Gay, R. Kirby, Constructing symplectic forms on 4-manifolds which vanish on circles, Geom. Topol. 8 (2004), 743-777.

[15] R. E. Gompf, A new construction of symplectic manifolds, Ann. Math. 142 (1995), 527-595.

[16] R.E. Gompf, A.I. Stipsicz, 4-manifolds and Kirby calculus, Graduate Studies in Math. 20, Amer. Math. Soc., Providence, 1999.

[17] M. Gromov, Pseudo-holomorphic curves in symplectic manifolds, Invent. Math. 82 (1985), 307-347.

[18] K. Honda, Transversality theorems for harmonic forms, Rocky Mountain J. Math. 34 (2004), 629-664.

[19] V. Kharlamov, V. Kulikov, On braid monodromy factorizations, Izv. Math. 67 (2003), 79-118.

[20] V. Kulikov, On a Chisini conjecture, Izv. Math. 63 (1999), 1139-1170.

[21] K. M. Luttinger, Lagrangian tori in $\mathbb{R}^{4}$, J. Differential Geom. 42 (1995) 220-228.

[22] B. Moishezon, Complex surfaces and connected sums of complex projective planes, Lecture Notes in Math. 603, Springer, Heidelberg, 1977.

[23] B. Moishezon, The arithmetic of braids and a statement of Chisini, Geometric Topology (Haifa, 1992), Contemp. Math. 164, Amer. Math. Soc., Providence, 1994, pp. 151-175.

[24] B. Ozbagci, A. Stipsicz, Noncomplex smooth 4-manifolds with genus 2 Lefschetz fibrations, Proc. Amer. Math. Soc. 128 (2000), 3125-3128.

[25] V. V. Shevchishin, On the local Severi problem, Int. Math. Res. Not. (2004), 211-237 (math.AG/0207048)

[26] B. Siebert, G. Tian, On the holomorphicity of genus two Lefschetz fibrations, preprint, to appear in Ann. Math (math.SG/0305343).

[27] C. H. Taubes, The Seiberg-Witten and the Gromov invariants, Math. Res. Lett. 2 (1995), 221-238.

[28] C. H. Taubes, The geometry of the Seiberg-Witten invariants, Surveys in Differential Geometry, Vol. III (Cambridge, 1996), Int. Press, Boston, 1998, pp. 299-339.

[29] C. H. Taubes, Seiberg-Witten invariants and pseudo-holomorphic subvarieties for selfdual, harmonic 2-forms, Geom. Topol. 3 (1999), 167-210.

[30] M. Teicher, Braid groups, algebraic surfaces and fundamental groups of complements of branch curves, Algebraic Geometry (Santa Cruz, 1995), Proc. Sympos. Pure Math., 62 (part 1), Amer. Math. Soc., Providence, 1997, pp. 127-150.

[31] W. Thurston, Some simple examples of symplectic manifolds, Proc. Amer. Math. Soc. 55 (1976), 467-468.

Department of Mathematics, M.I.T., Cambridge MA 02139, USA

E-mail address: auroux@math.mit.edu 